\title{
Investigation of All-photonic Multiple Signals Format Converter to Carrier- suppressed Signals
}

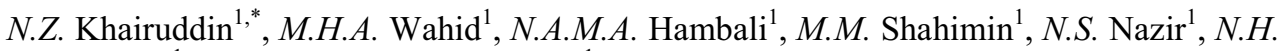 \\ Hasanuddin $^{1}$, and M. Muhammad. Ramli ${ }^{1}$ \\ ${ }^{1}$ Semiconductor Photonics \& Integrated Lightwave Systems (SPILS),School of Microelectronic \\ Engineering, Universiti Malaysia Perlis, Pauh Putra Campus, Malaysia.
}

\begin{abstract}
Advance modulation format such as carrier suppressed return to zero (CSRZ) is used in several optical communication systems. The generation of CSRZ signal is reported in this paper by means of allphotonics format converter to its suppressed carrier signal. It examines five photonics signals of NRZ, RZ, CRZ, soliton and duobinary. All formats are successfully converted to the carrier suppressed return to zero, CSRZ. The converter utilizes the semiconductor optical amplifier in a loop mirror and the control signal which is typically at ten times higher than the input signals. The suppressed signal can reduce the peak power alteration in the transmission. It also minimizes the nonlinear effects due to unavailability of the peak carrier. The CSRZ in the WDM system with 4 and 8 channels shows soliton as the best suppressed signal within selected parameters.
\end{abstract}

\section{Introduction}

In recent years, several modulation formats have been suggested as alternatives to the commonly used nonreturn-to-zero (NRZ) and return-to- zero (RZ) [1,2]. The main difference between conventional RZ and CSRZ is phase shift of $\pi$ between neighboring bits [1]. In its standard form CSRZ is generated by a single Mach-Zehnder modulator, driven by two sinusoidal waves at half the bit rate, and in phase opposition. An apparatus for generating a CSRZ signal is shown in some references [2,3]. The apparatus includes a mixer, a low pass filter, a driver amplifier and a single external modulator. The mixer generates a modulator input by mixing data with a half clock signal. The low pass filter band-limits the modulator input data into low frequency band data [3]. Several types of modulation formats are used in optical networks, and new type of formats are extensively studied and validated. To relief the potential of optical transmission systems and achieve higher transmission capacity, a lot of research on some special modulation formats other than traditional non return- to-zero (NRZ) and return-to-zero (RZ) has been done in recent years [4,5] including CSRZ [6].

\footnotetext{
* Corresponding author: zarhiedah@gmail.com
} 
This work proposes all-photonic multiple format converter to carrier suppressed signals for RZ-to-CSRZ, NRZ-to-CSRZ, CRZ-to-CSRZ, soliton-to-CSRZ and duobinary-to-CSRZ format conversion. Carrier suppressed return to zero reduces the main carrier component, which carries the majority of the signal power and narrows the optical spectrum to a format that has a higher spectral efficiency and more tolerant to chromatic dispersion. In WDM applications, CSRZ has been shown to be more sensitive, where there are carrier components of the transmitted signals. The higher the number of channels, the more of sensitivity of CSRZ. All optical signal processing including multiplexing, demultiplexing and format conversion have recently received much interest [1,6]. Next generation communication networks are a mixture situation where different technologies come to play due to the large variety of applications and to their hierarchal configuration that implies various specifications for the networks segments. All-optical format conversion is considered to be the main technology used in optical signal processing to enhance the flexibility of optical networks $[7,8]$.

\section{Principle operation \& setup}

Figure 1 shows the setup of the CSRZ converter. The conversion section is the main part which contains the nonlinear loop biased in between 0 to $2 \pi$. The control beam is half the data rate with sinusoidal property. As a result the refractive index also changes sinusoidally. If the signals injected into the loop propagating in opposite directions, both will interfere constructively or destructively that may end in transmission or reflection. This is governed by the phase shift between them which later converted to amplitude modulation. Hence, a sequence of alternating bits in phase is produced at the transmission port which is CSRZ signal.



Fig. 1. Simulation setup of the converter.

The signal power is derived from NRZ, RZ, CRZ, soliton and duobinary transmitters. This signal combines in a multiplexer with control beam where the power of both signals are controlled by attenuators. Both signals are then directed to an SOA for the modulation to take place. Eventually, both signals are coupled again and the CSRZ output is observed as depicted in Figure 2. All signals are again multiplexed up to 8 channels for verification its suitability in WDM networks. Details of the eye diagrams and optical power spectra are presented in the next section. 


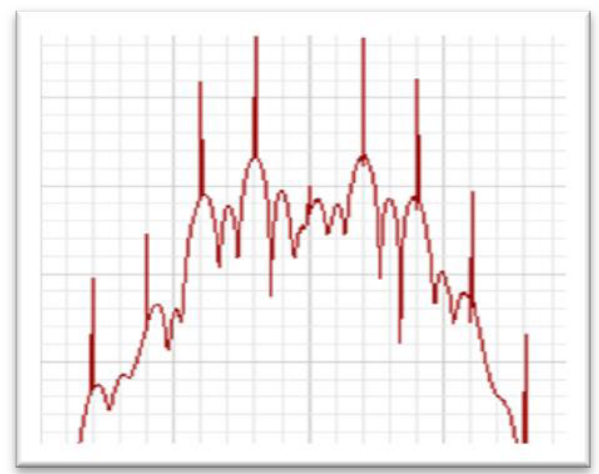

Fig. 2. Typical carrier suppressed spectrum.

\section{Results and discussion}

Table 1 summarizes the observation of the signals at both input and output ports. The signals are classified into original data and its converted eye and spectrum.

Table 1. Before and after conversion of each signal.

\begin{tabular}{|l|c|c|c|c|}
\hline \multirow{2}{*}{ Signal } & \multicolumn{2}{|c|}{ Before Conversion } & \multicolumn{2}{c|}{ After Conversion } \\
\hline NRZ & BER analysis & Spectrum & BER analysis \\
\hline RZ & & & \\
\hline CRZ & &
\end{tabular}

All signals are successfully converted with the same conversion parameters. The input power is maintained at $0 \mathrm{dBm}$ while the control power at $10 \mathrm{dBm}$. Injected current into the SOA is configured at $250 \mathrm{~mA}$ for $5 \mathrm{dBm}$ saturation output power. It is clearly observed that the carrier suppression denoted at the output. The level of suppression varies which is dependent on the powers and format of the signals. The RZ has shown to be the deepest 
carrier suppressed within the setup parameters. Changing these values might end up with different suppression ratio.

This work is extended to 4- and 8-channel WDM signals as depicted in Figure 3. Equal space between signals is used instead of random distance although it has similar possibility of conversion. It is found that the suppressed carrier signal easily generated. However, the control power needs an alteration with the number of channels increases. Table $2 \& 3$ depicts BER for back-to-back measurement of both WDM channels. Range of wavelengths are chosen from 1548 to $1555 \mathrm{~nm}$, while the control signal is kept at $1545 \mathrm{~nm}$. Some BERs are not recorded firmly due to the filtering of control power. When higher control is used, filtering selected wavelength also become more difficult. Thus, BER will give a small fluctuation in certain wavelength especially those closer to the control wavelength. Increasing the signal power will push the SOA into saturation that may also turns the BER to be worst. Properly adjusting powers and injected current could yield in better conversion.
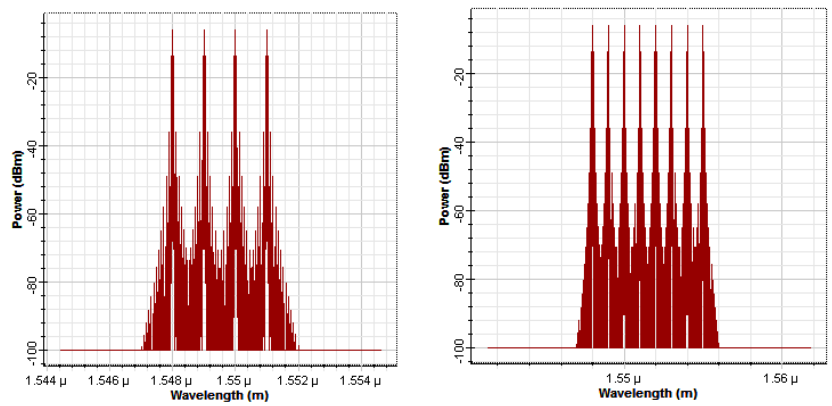

Fig. 3. Spectrum for 4-Channel and 8-Channel.

Table 2. BER for 4-channel.

\begin{tabular}{|l|l|l|l|l|l|}
\hline Wavelength & NRZ & RZ & CRZ & Soliton & Duobinary \\
\hline $1548 \mathrm{~nm}$ & 2.09 e-25 & 1.13 e-21 & 2.14 e-10 & 1.16 e-33 & 5.05 e-9 \\
\hline $1549 \mathrm{~nm}$ & 6.19 e-33 & 7.76 e-23 & 5.65 e-11 & 3.69 e-42 & 4.59 e-9 \\
\hline $1550 \mathrm{~nm}$ & 2.57 e-37 & 2.16 e-21 & 2.24 e-10 & 7.98 e-40 & 6.52 e-9 \\
\hline $1551 \mathrm{~nm}$ & 1.12 e-26 & 4.51 e-31 & 2.37 e-10 & 1.31 e-31 & 9.76 e-9 \\
\hline
\end{tabular}

Table 3. BER for 8-channel.

\begin{tabular}{|l|l|l|l|l|l|}
\hline Wavelength & NRZ & RZ & CRZ & Soliton & Duobinary \\
\hline 1548 & 2.67 e- 21 & 3.86 e-13 & 5.24 e- 21 & 3.54 e-48 & 2.59 e-10 \\
\hline 1549 & 7.21 e-16 & 2.01 e-13 & 1.06 e-12 & 4.67 e-35 & 2.14 e-9 \\
\hline 1550 & 1.45 e-16 & 1.87 e-13 & 8.40 e- 14 & 9.47 e-37 & 2.21 e- 8 \\
\hline 1551 & 1.22 e-22 & 1.22 e-13 & 6.71 e-27 & 7.58 e-47 & 6.29 e-9 \\
\hline 1552 & 1.39 e-19 & 2.71 e-13 & 2.14 e-17 & 2.05 e-30 & 5.01 e- 8 \\
\hline 1553 & 1.24 e-22 & 3.12 e-13 & 1.49 e-23 & 1.99 e-34 & 2.31 e-8 \\
\hline 1554 & 4.08 e-23 & 6.35 e-11 & 7.01 e-22 & 7.31 e-31 & 3.21 e-9 \\
\hline 1555 & 2.59 e- 22 & 3.71 e-11 & 1.66 e-19 & 1.67 e- 28 & 3.40 e-9 \\
\hline
\end{tabular}

Supressed carrier signals from its origin of soliton signal beat other formats in all observation. Duobinary has the poorest eye and spectrum that resulted to be the least converted signal. In order to confirm this remark, all signals have been put in the same multiplexer with the same configuration. The soliton supersedes other signal formats. This might be the nonlinear effect is reduced due to unavailability of the peak carrier in the signal 


\section{Conclusion}

All-optical format conversion is successfully performed using SOA in a nonlinear loop. Converted signals can be observed for NRZ, RZ, CRZ, soliton and duobinary. Carrier suppressed signal from soliton surpasses other formats with good BER at all wavelengths where asduobinary has shown the weakest likely to be converted. Multiple signals can be carrier supressed at the same time with one setup. Thus, the converter is suitable to be adopted in WDM networks.

The authors would like to thank the School of Microelectronics Engineering, Universiti Malaysia Perlis (UniMAP) especially SPILS for their support in this work. Partial of this research is funded by FRGS 9003-00461 under Ministry of Higher Education, Malaysia.

\section{References}

1. R. Ali, M.S Ali, T. Mir, B. Shabir, U.F. Lilla, Electrical and Electronics Engineering: An International Journal, 4, 35 (2015)

2. T. Tokle, C. Peucheret, P. Jeppesen, Opt. Commun., 225, 79 ( 2003)

3. D.S. Lee, M.S. Lee, S.S. Lee, J. SooKo, U.S. Patent 7412173 (2008)

4. Y. Yu, X. Zhang, D. Huang, Optics Express, 15, 1 (2007)

5. Prasenjit Pal, Optical fiber communication system-theory and analysis (online) http://www.gatewayforindia.com/technology/opticalfiber.htm.

6. M.H.A. Wahid, M.M. Nahas, R.A. Ibbotson, K. J. Blow, Opt. Commun., 332, 55 (2014)

7. A. Marculescu, S. Sygletos, J. Li, D. Karki, D. Hillerku\s s, S. Ben-Ezra, S. Tsadka, W. Freude, J. Leuthold, Optical Fiber Communication Conference (2009)

8. V. Sharma, A. Shrivastav, A. Jain, A. Panday, Int. J. Eng. Res. Appl., 2, 1619 (2012) 